\title{
A Systematic Review and Meta-Analysis of Randomised Controlled Trials, Contrasting the Safety Profile between Sodium-Glucose Cotransporter-2 Inhibitors and Placebo in Type 1 Diabetes Mellitus Patients
}

\author{
Sumanta Saha ${ }^{a}$ Sujata Saha ${ }^{b}$ \\ ${ }^{a}$ National Institute for Research in Tuberculosis, Chennai, India; ${ }^{b}$ Mankar College, Mankar, India
}

\section{Keywords}

Type 1 diabetes mellitus · Sodium-glucose cotransporter-2 inhibitor · Insulin · Side effects · Genital infection · Diabetic ketoacidosis

\begin{abstract}
Background: This study aims to contrast the side effects of treatment with sodium-glucose cotransporter-2 inhibitors (SGLT2i) and placebo in insulin-treated adult type 1 diabetes mellitus (T1DM) patients. Methods: Double-blinded randomized controlled trials that compare the above outcome were searched in electronic databases. Next, the risk of bias in eligible studies was assessed, and comparable outcomes were compared by a random-effects meta-analysis. Results: Seven eligible papers comprising about 3,900 participants were studied. All trials suffered from an unclear risk of detection bias and performance bias. In comparison with the placebo group, the risk of genital infection $(\mathrm{RR}=3.22, p<0.001$, $95 \% \mathrm{Cl} 2.31-4.49, R^{2}=0 \%$ ) and diabetic ketoacidosis (RR = $2.66, p=0.002,95 \% \mathrm{Cl} 1.45-4.89, I^{2}=0 \%$ ) was higher in the SGLT2i-treated group. Conclusion: SGLT2i treatment increased the risk of genital infection and diabetic ketoacidosis in adult insulin-treated T1DM patients.
\end{abstract}

(c) 2020 The Author(s)

Published by S. Karger AG, Basel

\section{Introduction}

The existing knowledge of the efficacy and safety of sodium-glucose cotransporter-2 inhibitors (SGLT2i) is primarily sourced from type 2 diabetes mellitus (T2DM) patients, for whom it is an established treatment and is endorsed by health authorities like the US Food and Drug Administration [1]. Examples of SGLT2i are Canagliflozin, dapagliflozin, empagliflozin, and ertugliflozin [2]. These drugs accelerate the elimination of glucose through the kidneys by inhibiting sodium-glucose cotransporter-2, a transport protein responsible for reabsorbing $90 \%$ of the filtered glucose $[3,4]$. In T2DM patients, SGLT2i help by reducing the blood glucose level, body weight, and blood pressure, and increasing the highdensity lipoprotein cholesterol [5].

Such benefits in T2DM patients have inspired SGLT2i use as an adjunct to insulin treatment in type 1 diabetes mellitus (T1DM) patients. Contemporary evidence from phase 2 and phase 3 trials on inhibitors of sodium-glucose cotransporter (as an adjunct to insulin treatment) in T1DM patients demonstrated a reduction in glycated haemoglobin levels, body weight, and everyday insulin dosage [6]. Based on the studies conducted on T2DM patients, some of the common side effects of these medications are urinary tract infections (UTIs), limb amputation, Fournier's gangrene, and genital infection [5,7-13].

Dr. Sumanta Saha

A-10/323

Kalyani, West Bengal 741235 (India)

sumanta.saha@uq.net.au 
Nevertheless, the safety profile of SGLT2i as an insulin accompaniment in T1DM patients is poorly understood [1]. A recent and relatively comprehensive systematic review and meta-analysis [14] that studied the side effects of SGLT2is in T1DM patients had potential pitfalls [15]; therefore, the evidence in this context remains poorly understood and needs additional reviews. Thus, this study aims to compare the above-listed side effects in T1DM patients treated with SGLT2i versus placebo.

\section{Methods}

The following were the inclusion criteria of this review: (1) clinical trials (of any duration) that were described as randomised, double-blinded, and parallel-arm (any number of arms); (2) study population must be adult (18 years or older) T1DM patients (irrespective of their estimated glomerular filtration rates with a glycated haemoglobin level of 7\% or more at screening, recruitment, or randomisation) who were on insulin treatment over the previous 12 months; (3) the following therapeutic interventions should have been compared as insulin adjuncts - daily SGLT2i supplementation versus placebo; (4) one of the following adverse outcomes must have been compared between the above intervention groups who received at least one dose of the test drug - UTIs, limb amputation, Fournier's gangrene, and genital infection. Outcome definitions and the follow-up time after which these side effects were reported, were accepted as per the trialists.

A trial was excluded from this review when: (1) study participants suffered from T2DM, gestational diabetes, or maturity-onset diabetes; (2) T1DM patients concurrently received any other antihyperglycaemic agent besides insulin and the trial drugs.

The following ancillary outcomes were also studied (when reported by the respective trials): severe hypoglycaemia, diabetic ketoacidosis (DKA), and study discontinuation due to treatment side effects. However, these did not contribute to the eligibility criteria. A pre-published protocol does not exist for this study. This review adheres to the PRISMA reporting guidelines [16].

Without any restriction to date and language, eligible trials (titles and abstracts) were searched in PubMed, Embase, and Scopus using the following search terms: "safety" OR "tolerance" OR "adverse event" OR "side effect" AND "canagliflozin" OR "dapagliflozin" OR "empagliflozin" OR "ertugliflozin" AND "type-1" OR "type1" OR "type 1" AND "diabetes" AND "trial" OR "clinical trial." Filters were used instead of the latter two search terms for Embase ("controlled clinical trial" and "randomized controlled trial") and PubMed ("clinical trial"). The last date of the search was July 7, 2019. Supplementary searches were conducted by skimming through the references of the included trials.

After eliminating the duplicate search results, the titles and abstracts of the remaining papers were read and matched against the eligibility criteria. Subsequently, full-text reading followed for articles that appeared to match the eligibility criteria or when a decision of inclusion or exclusion of a paper could not be made by reading the abstracts alone. Next, for each of the included studies, information pertinent to study design (including the description of participant consent and funding information), participants (diagnosis, total number randomised, mean age, gender distribution, eligibility criteria, and participant nationality), compared interventions, and outcomes of interest were extracted. The entire database search and the data extraction process were performed by the first author and then checked by the co-author for errors. The authors mitigated any disagreement by discussion.

Then, the risk of bias in the trials was assessed independently by the authors for selection bias, performance bias, detection bias, attrition bias, reporting bias, and other types of bias (using the Cochrane Collaboration's tool) [17]. The appraisal findings of the authors were collated, and in case of a mismatch, a discourse ensued.

Meta-analysis was performed to compare the risk ratio of the individual adverse effects between SGLT2i therapy and placebo treatment. For a meta-analytic comparison with placebo, respective outcome data were aggregated when different dosages of a particular SGLT2i were tested in a trial. Since the trial population originated from different parts of the globe and were treated in diverse treatment settings, clinical heterogeneity was assumed across the trials. Therefore, we used a random-effects model (DerSimonian and Laird method) to estimate the summary effects. The predictive intervals were determined for an outcome when at least three studies were available for meta-analysis. We added 0.5 to each cell of the $2 \times 2$ table when an adverse event did not occur in any one of the compared intervention groups (of a trial). For any particular outcome, a trial was excluded from the meta-analysis if an adverse event did not happen in both of the treatment groups. Heterogeneity assessment included the $p$ value of Cochrane's $Q$ $\left(<0.1\right.$ denoting statistical significance) in conjunction with the $I^{2}$ statistics. When the percentages of $I^{2}$ values were $0-40,30-60,50-$ 90 , and $75-100$, they represented less, moderate, substantial, and considerable chances of heterogeneity, respectively [17]. Publication bias was visually assessed using funnel plots. For the respective outcomes, the sensitivity analyses included a repetition of the meta-analysis using a fixed-effects model and subsequently by dropping one study each time. The statistical significance of summary estimates was determined at a $p$ value $<0.05$. For all statistical analyses, we used Stata statistical software (StataCorp, College Station, TX, USA).

\section{Results}

The database search produced 73 papers. After excluding the duplicates, the titles and abstracts of 40 papers were read. Out of the 14 papers that were read in full, 7 papers matching the eligibility criteria were included in this review (Fig. 1). The reviewed trials sourced data from about 3,900 participants (from almost 77 nations) [1824]. Three trials contrasted the side effects of three different doses of empagliflozin $(2.5,10$, and $25 \mathrm{mg}$ ) and placebo [18-20]. Three trials studied the adverse effects of dapagliflozin (and placebo), among which two [21, 24] included the 5- and 10-mg doses while the remaining one tested the 1 and $2.5 \mathrm{mg}$ dosage, additionally [23]. One trial compared the harmful effects of 100 and $300 \mathrm{mg}$ of canagliflozin and placebo [22]. The side effects of ertugliflozin in T1DM patients were not studied in any of the 
Fig. 1. PRISMA 2009 flow diagram (from Moher et al. [28]).

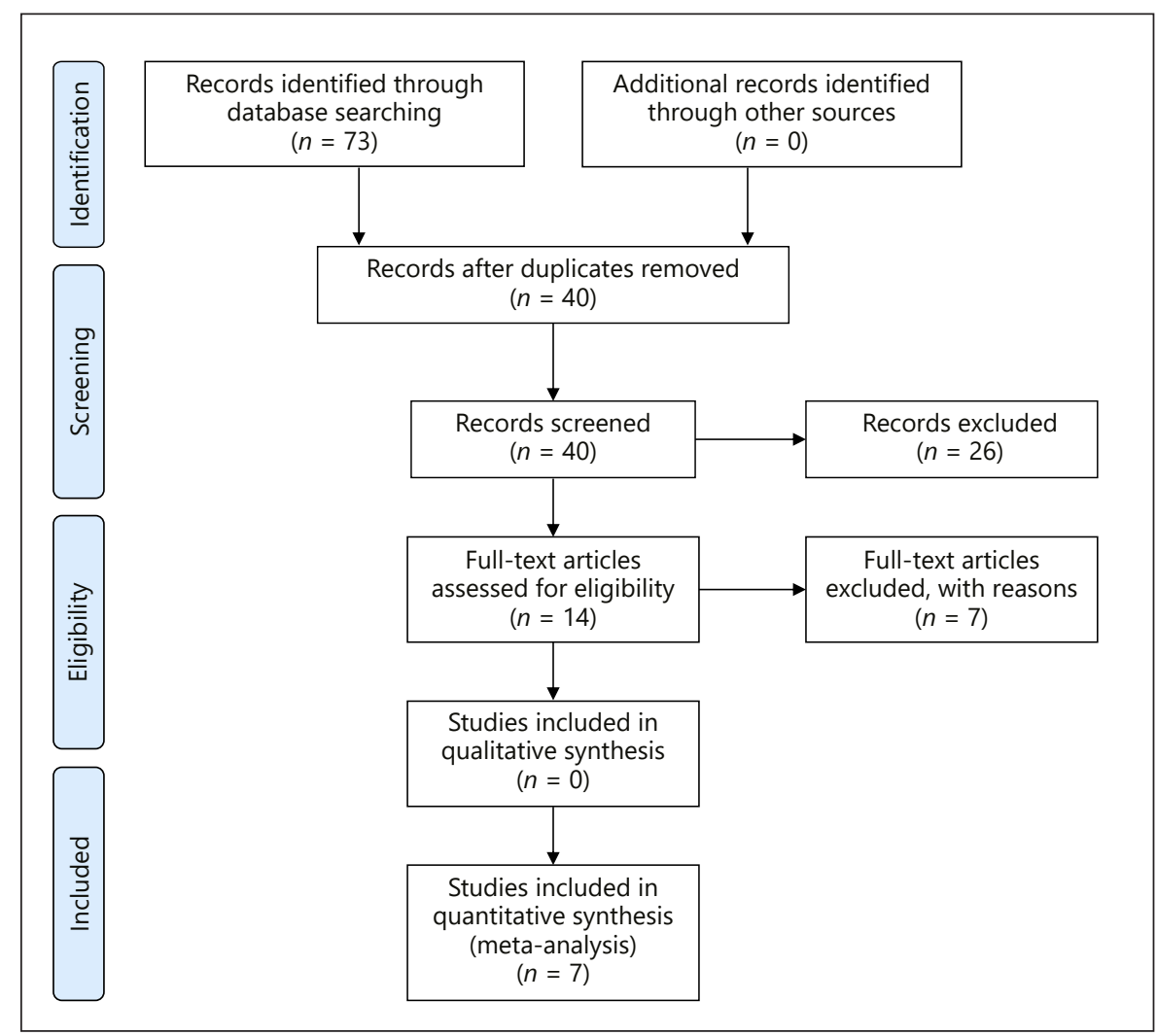

trials. Table 1 depicts the salient features of the trials. All trials obtained participant consent and provided funding information [18-24].

Only the study by Rosenstock et al. [19] sourced data from two trials and presented the combined side effects for placebo and 10 and $25 \mathrm{mg}$ empagliflozin. The respective outcomes for $2.5 \mathrm{mg}$ empagliflozin were reported in one of these trials [19]. For the meta-analysis, the combined placebo outcome data (of these trials) for the respective outcomes were compared with the aggregated outcome data of the different dosages of empagliflozin [19].

All trials were at an unclear risk of selection bias (except for the study by Pieber et al. [20]), performance bias, and detection bias [18-24]. These trials were at a low risk of bias for reporting bias and attrition bias [18-24]. The details of the risk of bias assessment are depicted in Table 1.

The adverse outcome, Fournier's gangrene, was not reported in any of the papers. Only in one trial, in the 2.5 mg empagliflozin-treated group, a minor toe amputation was reported in a patient who had a previous history of amputations and peripheral arterial disease [19]. For the remaining side effects, the forest plots in Figure 2a-e present the outcome data along with the summary estimates and confidence intervals (CI).
Compared to the placebo, SGLT2i treatment increased the risk of developing genital infection $(\mathrm{RR}=3.22, p<$ $0.001,95 \%$ CI $2.31-4.49, \chi^{2}$ test $p$ value $\left.=0.64, I^{2}=0 \%\right)$ and DKA $\left(\mathrm{RR}=2.66, p=0.002,95 \%\right.$ CI $1.45-4.89, \chi^{2}$ test $p$ value $\left.=0.405, I^{2}=0 \%\right)$ in T1DM patients. While the predictive interval (2.02-5.15) for genital infection suggested that future trials are unlikely to observe a different finding, that for DKA $(0.07-10.11)$ hinted of a plausible protective role of SGLT2i in forthcoming trials.

The risk of the following adverse outcomes did not vary between the SGLT2i-treated group and the placebo group: severe hypoglycaemia $(\mathrm{RR}=1.07, p=0.777,95 \%$ CI $0.66-1.74, \chi^{2}$ test $p$ value $\left.=0.514, I^{2}=0 \%\right)$, study discontinuation due to treatment side effects $(\mathrm{RR}=1.40, p=$ $0.080,95 \%$ CI $0.96-2.03, \chi^{2}$ test $p$ value $\left.=0.933, I^{2}=0 \%\right)$, and UTI $\left(\mathrm{RR}=1.04, p=0.773,95 \%\right.$ CI $0.81-1.34, \chi^{2}$ test $p$ value $\left.=0.685, I^{2}=0 \%\right)$. For all outcomes that were compared in the meta-analysis, the findings were homogenous across all trials. Visual inspection of funnel plots suggested an absence of publication bias (plots not shown). On sensitivity analysis, the findings for the respective outcomes neither changed on using a fixed-effects model (Fig. 2) nor on dropping one study at a time and repeating the meta-analysis (Table 2 ).
Saha/Saha 


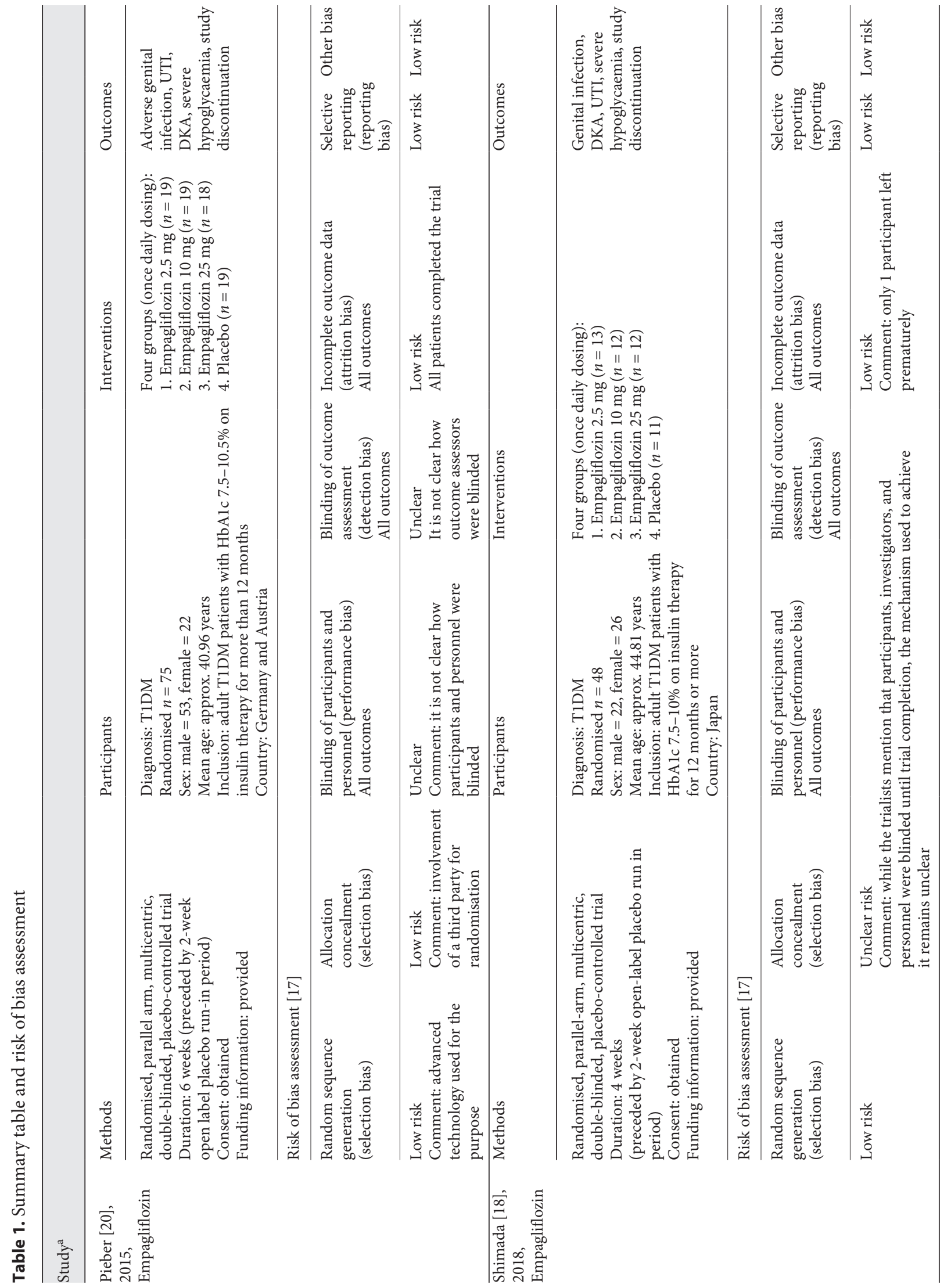




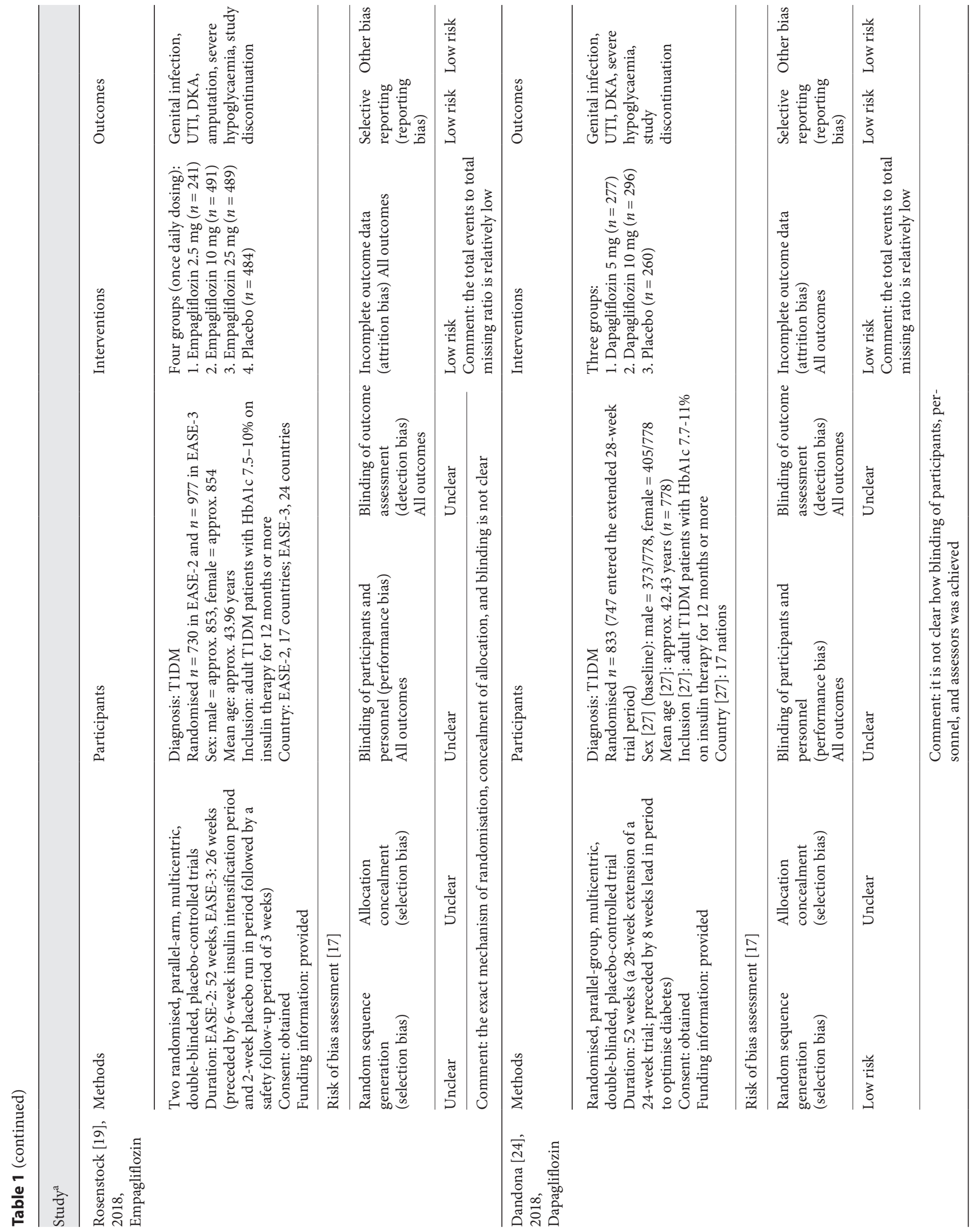



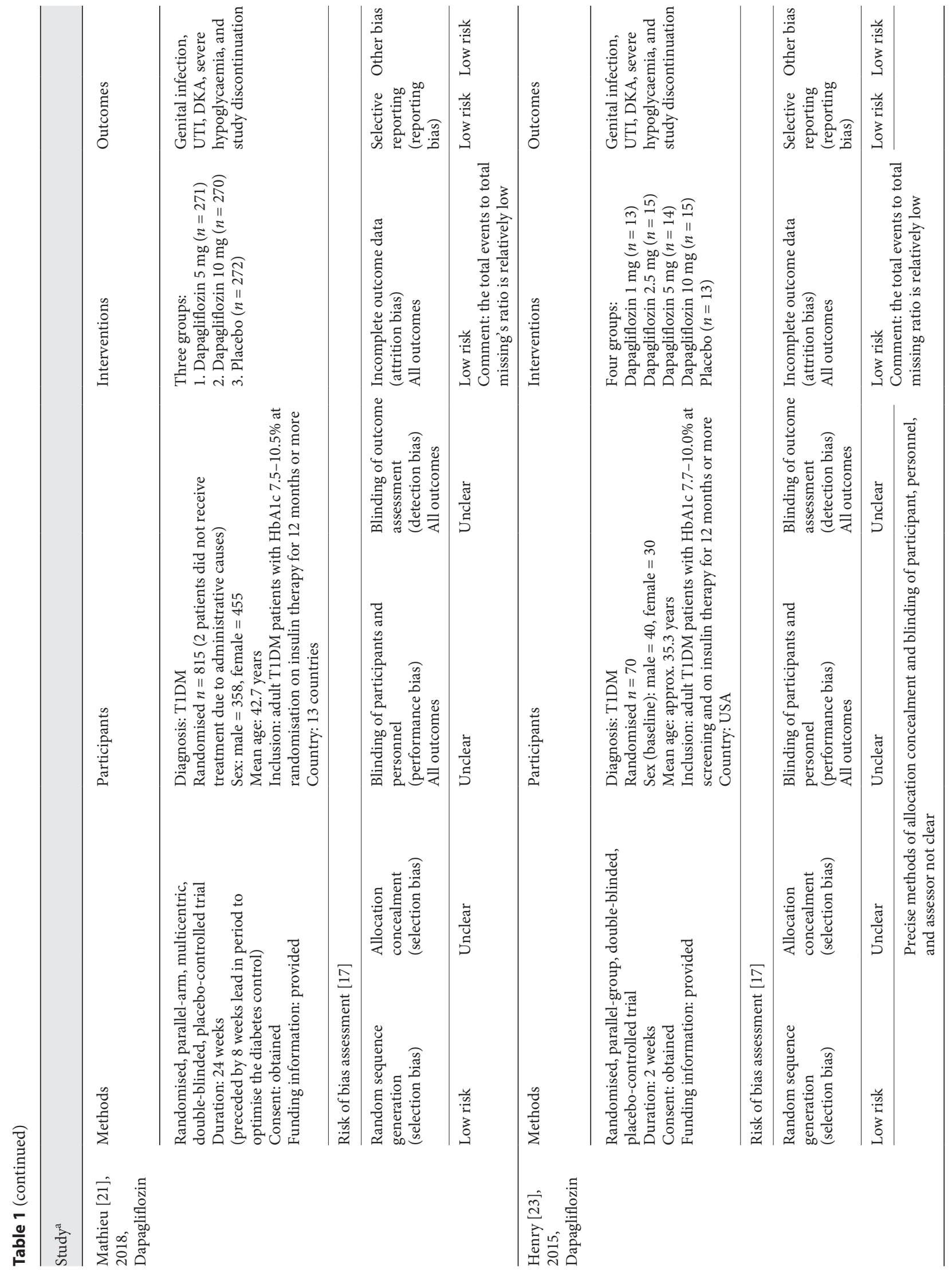


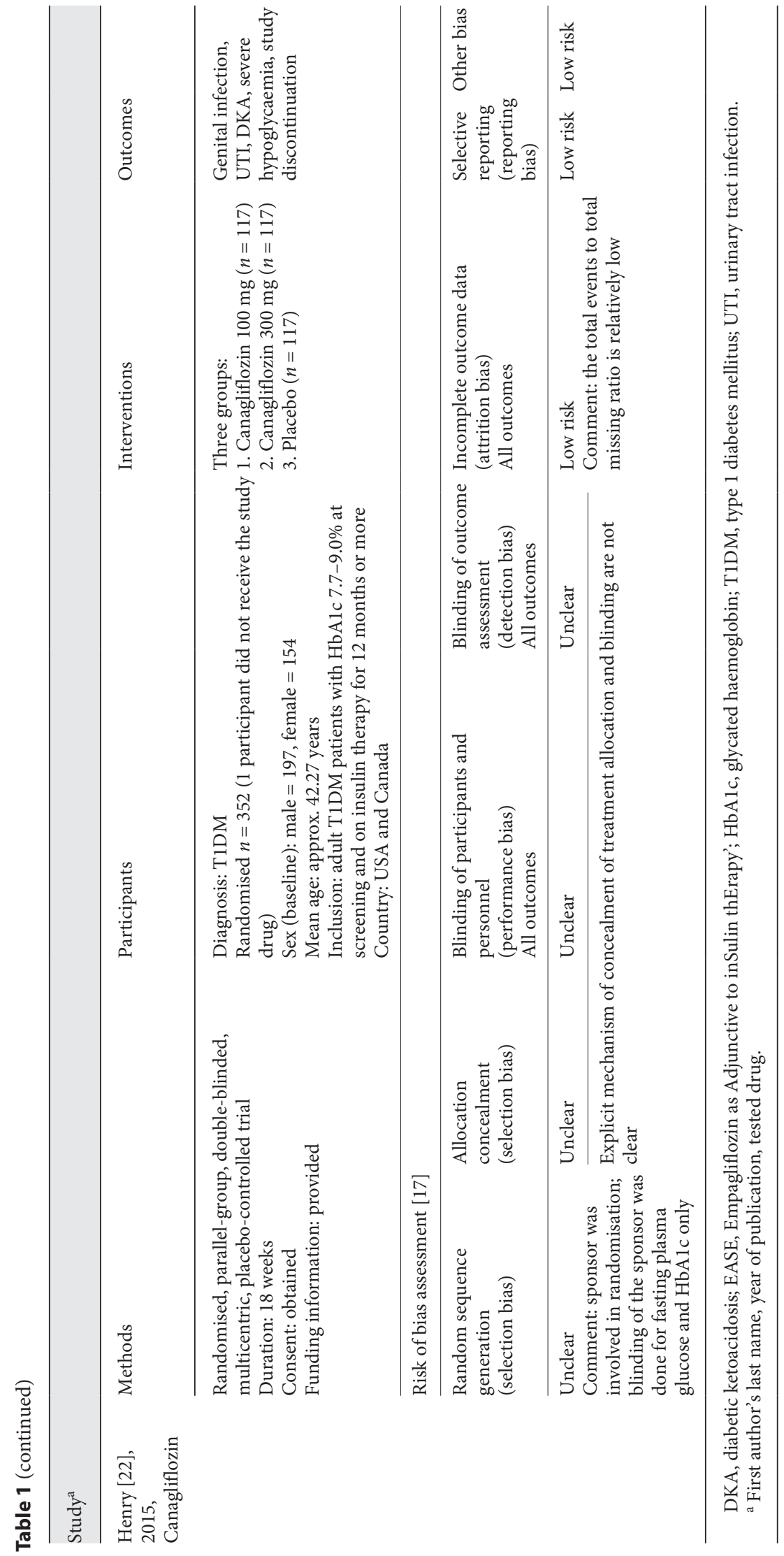




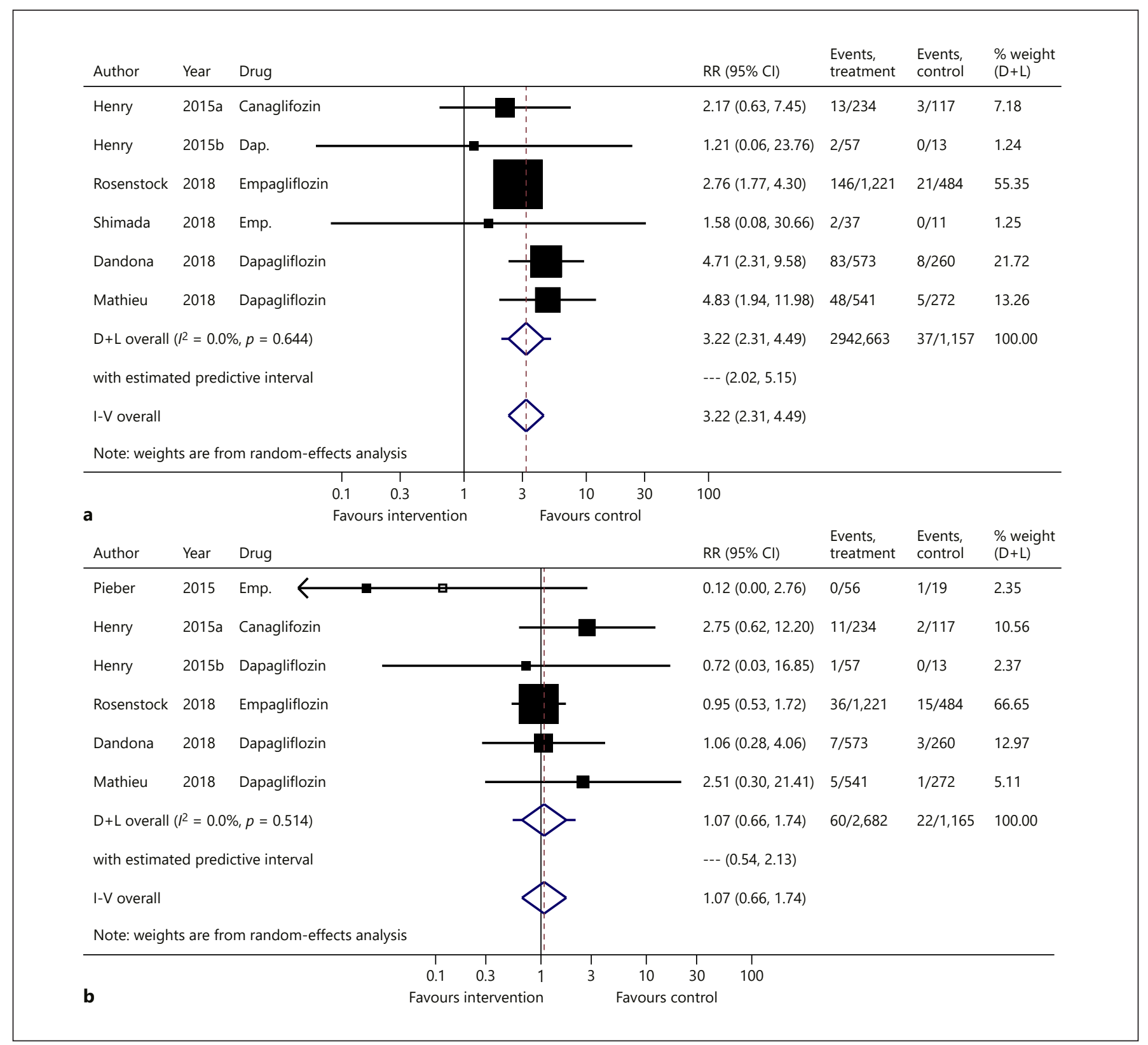

Fig. 2. Forest plots comparing various outcomes: genital infection (a); severe hypoglycaemia (b); study discontinuation due to adverse effects (c); UTI (d); DKA (e).

(Figure continued on next page.)

\section{Discussion}

The concurrent treatment with SGLT2i and insulin in T1DM patients increased the risk of acquiring genital infection and DKA, and the finding was consistent across the trials despite the geographically diverse trial population (involving about 3,900 participants from 77 nations) and settings. While the trials chiefly had an unclear risk of selection bias, detection bias, and performance bias, the risk of reporting bias and attribution bias were consistently low among them.

Using the GRADE system by the GRADE Working Group (2004) [25], the quality of evidence for the genital infection and DKA outcomes was downgraded by one level and rated as moderate-quality evidence due to the unclear risk of biases present among the trials. 


\begin{tabular}{|c|c|c|c|c|c|c|}
\hline Author & Year & Drug & $\mathrm{RR}(95 \% \mathrm{Cl})$ & $\begin{array}{l}\text { Events, } \\
\text { treatment }\end{array}$ & $\begin{array}{l}\text { Events, } \\
\text { control }\end{array}$ & $\begin{array}{l}\% \text { weight } \\
(D+L)\end{array}$ \\
\hline Henry & $2015 a$ & Canaglifozin & $3.51(0.18,67.49)$ & $3 / 234$ & $0 / 117$ & 1.60 \\
\hline Henry & $2015 b$ & Dap. & $0.72(0.03,16.85)$ & $1 / 57$ & $0 / 13$ & 1.41 \\
\hline Rosenstock & 2018 & Empagliflozin & $1.56(0.87,2.77)$ & $55 / 1,221$ & $14 / 484$ & 41.94 \\
\hline Dandona & 2018 & Dapagliflozin & $1.21(0.57,2.57)$ & $24 / 573$ & $9 / 260$ & 24.71 \\
\hline Mathieu & 2018 & Dapagliflozin & $1.33(0.67,2.61)$ & $29 / 541$ & $11 / 272$ & 30.34 \\
\hline \multicolumn{3}{|c|}{$\mathrm{D}+\mathrm{L}$ overall $\left(R^{2}=0.0 \%, p=0.933\right)$} & $1.40(0.96,2.03)$ & $112 / 2,626$ & $34 / 1,146$ & 100.00 \\
\hline \multicolumn{3}{|c|}{ with estimated predictive interval } & $---(0.76,2.56)$ & & & \\
\hline
\end{tabular}

I-V overall

Note: weights are from random-effects analysis

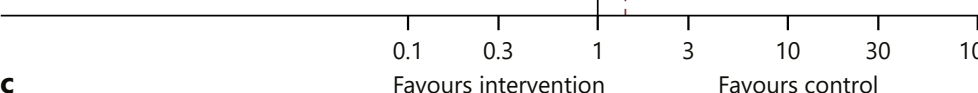

C Favours intervention

00

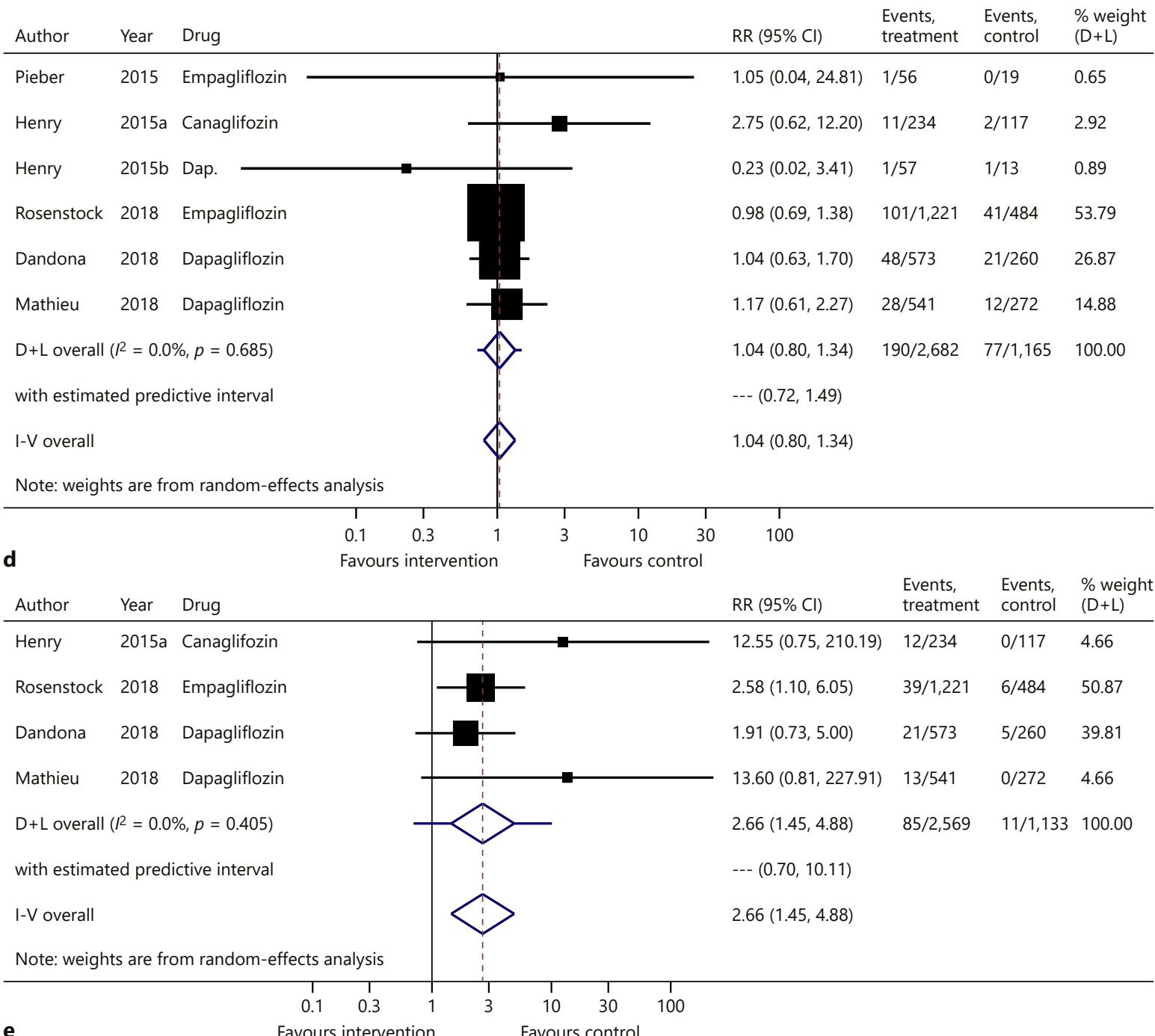


Table 2. Sensitivity analysis

\begin{tabular}{|c|c|c|c|c|c|}
\hline \multirow[t]{2}{*}{ Outcome } & \multirow[t]{2}{*}{ Excluded trial } & \multicolumn{2}{|c|}{ Effect size } & \multirow[t]{2}{*}{$p$ value } & \multirow[t]{2}{*}{$I^{2}, \%$} \\
\hline & & $\mathrm{FE}$ & $\mathrm{RE}$ & & \\
\hline \multirow[t]{6}{*}{ Genital infection } & Shimada [18], 2018 & 3.25 & 3.25 & 0.000 & 0.0 \\
\hline & Rosenstock [19], 2018 & 3.91 & 3.91 & 0.000 & 0.0 \\
\hline & Dandona [24], 2018 & 2.90 & 2.90 & 0.000 & 0.0 \\
\hline & Mathieu [21], 2018 & 3.03 & 3.03 & 0.000 & 0.0 \\
\hline & Henry [23], 2015 & 3.26 & 3.26 & 0.000 & 0.0 \\
\hline & Henry [22], 2015 & 3.32 & 3.32 & 0.000 & 0.0 \\
\hline \multirow[t]{6}{*}{ Severe hypoglycemia } & Pieber [20], 2015 & 1.13 & 1.13 & 0.622 & 0.0 \\
\hline & Rosenstock [19], 2018 & 1.36 & 1.36 & 0.469 & 0.0 \\
\hline & Dandona [24], 2018 & 1.08 & 1.11 & 0.742 & 5.8 \\
\hline & Mathieu [21], 2018 & 1.02 & 1.02 & 0.924 & 0.0 \\
\hline & Henry [23], 2015 & 1.08 & 1.10 & 0.724 & 4.4 \\
\hline & Henry [22], 2015 & 0.96 & 0.96 & 0.875 & 0.0 \\
\hline \multirow[t]{5}{*}{ Discontinuation from study } & Rosenstock [19], 2018 & 1.29 & 1.29 & 0.308 & 0.0 \\
\hline & Dandona [24], 2018 & 1.46 & 1.46 & 0.083 & 0.0 \\
\hline & Mathieu [21], 2018 & 1.43 & 1.43 & 0.119 & 0.0 \\
\hline & Henry [23], 2015 & 1.41 & 1.41 & 0.074 & 0.0 \\
\hline & Henry [22], 2015 & 1.38 & 1.38 & 0.097 & 0.0 \\
\hline \multirow[t]{6}{*}{ UTI } & Pieber [20], 2015 & 1.04 & 1.04 & 0.774 & 0.0 \\
\hline & Rosenstock [19], 2018 & 1.12 & 1.12 & 0.569 & 0.0 \\
\hline & Dandona [24], 2018 & 1.04 & 1.04 & 0.803 & 0.0 \\
\hline & Mathieu [21], 2018 & 1.02 & 1.02 & 0.908 & 0.0 \\
\hline & Henry [23], 2015 & 1.05 & 1.05 & 0.695 & 0.0 \\
\hline & Henry [22], 2015 & 1.01 & 1.01 & 0.950 & 0.0 \\
\hline \multirow[t]{4}{*}{$\mathrm{DKA}$} & Rosenstock [19], 2018 & 2.75 & 3.93 & 0.051 & 31.2 \\
\hline & Henry [23], 2015 & 2.46 & 2.46 & 0.005 & 0.0 \\
\hline & Dandona [24], 2018 & 3.31 & 3.60 & 0.007 & 7.2 \\
\hline & Mathieu [21], 2018 & 2.46 & 2.46 & 0.005 & 0.0 \\
\hline
\end{tabular}

DKA, diabetic ketoacidosis; FE, fixed-effects model meta-analysis; RE, random-effects model meta-analysis; UTI, urinary tract infection.

Then, we compared the findings of this paper with those conducted on T2DM patients (in the same context). Similar to the findings of this study, a meta-analysis of randomised controlled trials on T2DM patients comparing the adverse treatment outcomes between SGLT2i and placebo found an increased risk of genital infection and no difference in the risk of severe hypoglycaemia and UTI [5]. On the other hand, unlike the findings of our study, a meta-analysis of clinical trials on T2DM patients did not find any difference in the risk of DKA between these interventions [26]. However, as suggested by the predictive interval (mentioned above), we cannot rule out with absolute certainty that future trials will not find SGLT2i beneficial in decreasing the incidence of DKA in insulintreated T1DM patients compared to the placebo.
Next, we mention the implications of this study. Institutions responsible for legalising medicines may find this research useful in understanding the contemporary evidence and its rigor on the safety profile of SGLT2i in comparison with placebo in T1DM patients. Additionally, this study suggests that ertugliflozin's safety profile in T1DM patients remains unexplored; henceforth, in future trialists may consider the possibility of comparing its adverse outcomes with placebo. Likewise, only one clinical trial tested the safety of canagliflozin in T1DM patients [22], which highlights the requirement of newer trials to understand the overall safety profile of SGLT2i better. Finally, from our study, care providers of T1DM patients may gain some insight regarding the safety profile of SGLT2i. 
Here we state the strengths of this paper. First, it provides a dose-irrespective overview of adversities associated with SGLT2i in T1DM patients. Next, the findings of this study are likely to be rigorous, since it includes data from studies that are considered as the highest level of epidemiological evidence (i.e., double-blinded randomized controlled trials) and the sensitivity analysis replicates the findings of the preliminary analysis. Third, this review is likely to be more comprehensive as the database search process was not restricted to any date or language and included trials of any duration. Lastly, excluding the trials that used any anti-hyperglycaemic drugs other than insulin and SGLT2i allowed us to produce evidence specific to SGLT2i therapy in T1DM patients as an insulin adjunct.

Nevertheless, despite these strengths, this review has a few weaknesses. At the outcome level, the risk of performance bias and detection bias was unclear across all studies. Besides, as this study included only parallel-arm randomized controlled trials, incorporating evidence from other study designs like crossover studies or sound observational studies was beyond the scope of this review. Moreover, because of limited resources, we could not search for various other reputed databases like Web of Science.

\section{Conclusion}

Juxtaposed to placebo, SGLT2i increase the risk of genital infection and DKA in insulin-treated adult TIDM patients.

\section{Statement of Ethics}

As no human subjects were involved in this paper, ethical approval was not needed.

\section{Disclosure Statement}

The authors have no conflicts of interest to declare. This paper was produced by the authors independently and not related to their affiliated institutions.

\section{Funding Sources}

This study did not receive any funding.

\section{References}

1 US Food and Drug Administration. Sodiumglucose Cotransporter-2 (SGLT2) Inhibitors [Internet]. 2018. Available from: https:// www.fda.gov/drugs/postmarket-drug-safetyinformation-patients-and-providers/sodium-glucose-cotransporter-2-sglt2-inhibitors

2 New drug: Ertugliflozin for type 2 diabetes. Aust Prescr. 2019;42(2):70-2.

3 Boeder S, Edelman SV. Sodium-glucose cotransporter inhibitors as adjunctive treatment to insulin in type 1 diabetes: a review of randomized controlled trials. Diabetes Obes Metab. 2019 Apr;21(S2 Suppl 2):62-77.

4 Davidson JA. SGLT2 inhibitors in patients with type 2 diabetes and renal disease: overview of current evidence. Postgrad Med. 2019 May;131(4):251-60.

5 Monami M, Nardini C, Mannucci E. Efficacy and safety of sodium glucose co-transport-2 inhibitors in type 2 diabetes: a meta-analysis of randomized clinical trials. Diabetes Obes Metab. 2014 May;16(5):457-66.

6 McCrimmon RJ, Henry RR. SGLT inhibitor adjunct therapy in type 1 diabetes. Diabetologia. 2018 Oct;61(10):2126-33.

7 US Food and Drug Administration. FDA Drug Safety Communication: FDA confirms increased risk of leg and foot amputations with the diabetes medicine canagliflozin (Invokana, Invokamet, Invokamet XR) [Internet]. 2017. Available from: https://www.fda. gov/drugs/drug-safety-and-availability/fdadrug-safety-communication-fda-confirmsincreased-risk-leg-and-foot-amputations-diabetes-medicine

8 US Food and Drug Administration. FDA Drug Safety Communication: FDA revises label of diabetes drug canagliflozin (Invokana, Invokamet) to include updates on bone fracture risk and new information on decreased bone mineral density [Internet]. 2016. Available from: https://www.fda.gov/drugs/drugsafety-and-availability/fda-drug-safety-communication-fda-revises-label-diabetes-drugcanagliflozin-invokana-invokamet

9 Hsia DS, Grove O, Cefalu WT. An update on sodium-glucose co-transporter-2 inhibitors for the treatment of diabetes mellitus. Curr Opin Endocrinol Diabetes Obes. 2017 Feb; 24(1):73-9.

10 US Food and Drug Administration. FDA Drug Safety Communication: FDA strengthens kidney warnings for diabetes medicines canagliflozin (Invokana, Invokamet) and dapagliflozin (Farxiga, Xigduo XR) [Internet]. 2016. Available from: https://www.fda. gov/drugs/drug-safety-and-availability/fdadrug-safety-communication-fda-strengthens-kidney-warnings-diabetes-medicinescanagliflozin

11 US Food and Drug Administration. FDA Drug Safety Communication: FDA revises la- bels of SGLT2 inhibitors for diabetes to include warnings about too much acid in the blood and serious urinary tract infections [Internet]. 2018. Available from: https://www. fda.gov/drugs/drug-safety-and-availability/ fda-drug-safety-communication-fda-reviseslabels-sglt2-inhibitors-diabetes-includewarnings-about

12 US Food and Drug Administration. FDA warns about rare occurrences of a serious infection of the genital area with SGLT2 inhibitors for diabetes [Internet]. 2018. Available from: https://www.fda.gov/drugs/drug-safety-and-availability/fda-warns-about-rare-occurrences-serious-infection-genital-areasglt2-inhibitors-diabetes

13 Scheen AJ. An update on the safety of SGLT2 inhibitors. Expert Opin Drug Saf. 2019 Apr; 18(4):295-311.

14 Yamada T, Shojima N, Noma H, Yamauchi T, Kadowaki T. Sodium-glucose co-transporter-2 inhibitors as add-on therapy to insulin for type 1 diabetes mellitus: systematic review and meta-analysis of randomized controlled trials. Diabetes Obes Metab. 2018 Jul;20(7):1755-61.

15 Saha S. An appraisal of a systematic review and meta-analysis of randomized clinical trials on the efficacy and safety of sodium-glucose cotransporter- 2 inhibitors as an adjunct to insulin therapy in type 1 diabetes atients. Int J Diabetes Metab. doi: 10.1159/000502743. 
16 Liberati A, Altman DG, Tetzlaff J, Mulrow C, Gøtzsche PC, Ioannidis JP, et al. The PRISMA statement for reporting systematic reviews and meta-analyses of studies that evaluate health care interventions: explanation and elaboration. J Clin Epidemiol. 2009 Oct; 62(10):e1-34.

17 Higgins J, Churchill R, Chandler J, Cumpston $\mathrm{M}$, editors. Cochrane handbook for systematic reviews of interventions version 5.2.0 (updated June 2017). Chichester: Wiley; 2017.

18 Shimada A, Hanafusa T, Yasui A, Lee G, Taneda Y, Sarashina A, et al. Empagliflozin as adjunct to insulin in Japanese participants with type 1 diabetes: results of a 4-week, double-blind, randomized, placebo-controlled phase 2 trial. Diabetes Obes Metab. 2018 Sep; 20(9):2190-9.

19 Rosenstock J, Marquard J, Laffel LM, Neubacher D, Kaspers S, Cherney DZ, et al. Empagliflozin as adjunctive to insulin therapy in type 1 diabetes: the EASE trials. Diabetes Care. 2018 Dec;41(12):2560-9.

20 Pieber TR, Famulla S, Eilbracht J, Cescutti J, Soleymanlou N, Johansen OE, et al. Empagliflozin as adjunct to insulin in patients with type 1 diabetes: a 4-week, randomized, place- bo-controlled trial (EASE-1). Diabetes Obes Metab. 2015 Oct;17(10):928-35.

21 Mathieu C, Dandona P, Gillard P, Senior P, Hasslacher C, Araki E, et al.; DEPICT-2 Investigators. Efficacy and safety of dapagliflozin in patients with inadequately controlled type 1 diabetes (the DEPICT-2 Study): 24-week results From a randomized controlled trial. Diabetes Care. 2018 Sep;41(9): 1938-46.

22 Henry RR, Thakkar P, Tong C, Polidori D, Alba M. Efficacy and safety of canagliflozin, a sodium-glucose cotransporter 2 inhibitor, as add-on to insulin in patients with type 1 diabetes. Diabetes Care. 2015a Dec;38(12):225865.

23 Henry RR, Rosenstock J, Edelman S, Mudaliar S, Chalamandaris AG, Kasichayanula S, et al. Exploring the potential of the SGLT2 inhibitor dapagliflozin in type 1 diabetes: a randomized, double-blind, placebo-controlled pilot study. Diabetes Care. 2015b Mar;38(3): 412-9.

24 Dandona P, Mathieu C, Phillip M, Hansen L, Tschöpe D, Thorén F, et al.; DEPICT-1 Investigators. Efficacy and Safety of Dapagliflozin in Patients With Inadequately Controlled
Type 1 Diabetes: The DEPICT-1 52-Week Study. Diabetes Care. 2018 Dec;41(12):25529.

25 Atkins D, Best D, Briss PA, Eccles M, FalckYtter Y, Flottorp S, et al.; GRADE Working Group. Grading quality of evidence and strength of recommendations. BMJ. 2004 Jun; 328(7454): 1490.

26 Monami M, Nreu B, Zannoni S, Lualdi C, Mannucci E. Effects of SGLT-2 inhibitors on diabetic ketoacidosis: a meta-analysis of randomised controlled trials. Diabetes Res Clin Pract. 2017 Aug;130:53-60.

27 Dandona P, Mathieu C, Phillip M, Hansen L, Griffen SC, Tschöpe D, et al. Efficacy and safety of dapagliflozin in patients with inadequately controlled type 1 diabetes (DEPICT-1): 24 week results from a multicentre, double-blind, phase 3, randomised controlled trial. Lancet Diabetes Endocrinol. 2017;5: 864-76.

28 Moher D, Liberati A, Tetzlaff J, Altman DG; PRISMA Group. Preferred reporting items for systematic reviews and meta-analyses: The PRISMA Statement. PLoS Med. 2009; 6(7):e1000097. Doi: 10.1371/journal.pmed 1000097) 